\title{
MicroRNA-144 attenuates cardiac ischemia/reperfusion injury by targeting FOXO1
}

\author{
LUSHA E $^{1,2}$, HONG JIANG ${ }^{1}$ and ZHIBING LU ${ }^{1}$ \\ ${ }^{1}$ Department of Cardiology, Renmin Hospital of Wuhan University, Hubei General Hospital, Wuchang, Wuhan, Hubei 430060; \\ ${ }^{2}$ Cardiology Department, Inner Mongolia People's Hospital, Hohhot, Inner Mongolia 001017, P.R. China
}

Received September 8, 2017; Accepted February 22, 2018

DOI: $10.3892 /$ etm.2019.7161

\begin{abstract}
Cardiovascular ischemic disease refers to a large class of conditions that are harmful to human health. A number of previous studies have demonstrated that microRNAs (miRs) have notable roles in regulating cardiac injury. miR-144 is influential in the differentiation, growth, and metastatic processes of cells; however, the impact of miR-144 in cardiac ischemia/reperfusion (I/R) injury has not been thoroughly elucidated to date. In the present study, reverse transcription quantitative polymerase chain reaction was used to evaluate RNA expression. In addition, TTC staining was performed to detect the infarct area of the ischemic myocardia and a terminal deoxynucleotidyl-transferase-mediated dUTP nick end labeling assay was utilized to detect the apoptosis of the myocardia. It was observed that miR-144 expression is downregulated in an $\mathrm{I} / \mathrm{R}$ model in rats and that overexpression of miR-144 significantly reduced myocardial ischemic injury and apoptosis. Consistent with this result, similar findings were demonstrated in $\mathrm{H} 9 \mathrm{c} 2$ cells subjected to hypoxia/reoxygenation. Bioinformatic analysis using MiRanda and TargetScan, and luciferase assays confirmed that forkhead box protein O1was the target of miR-144. These findings suggest that miR-144 may be exploited as a novel molecular marker or therapeutic target for myocardial I/R injury.
\end{abstract}

\section{Introduction}

The 'gold standard' for treatment of myocardial infarction is to immediately reperfuse the occluded coronary artery (1). However, this treatment leads to myocardial ischemia/reperfusion (I/R) injury (MIR), in which blood perfusion has been restored following a long period of ischemia and results in such dysfunctions as systolic function decline, coronary flow and vascular reactivity variations (2). As MIR serves an important

Correspondence to: Dr Zhibing Li, Department of Cardiology, Renmin Hospital of Wuhan University, Hubei General Hospital, 238 Jiefang Road, Wuchang, Wuhan, Hubei 430060, P.R. China E-mail: luzhibingzb@126.com

Key words: microRNA-144, forkhead box protein O1, ischemia/ reperfusion, myocardium role in developing heart diseases, the mechanism of MIR has been widely investigated (3-5). It is a complex phenomenon and maybe caused by calcium overload, endothelial cell activation, mitochondrial damage, the formation of reactive oxygen species (ROS), dysregulated vascular relaxation and white cell plug formation (6). The treatment for MIR is limited due to the lack of comprehensive understanding of the pathological process during ischemia/reperfusion (I/R) $(7,8)$. Therefore, there is a pressing need to elucidate the underlying mechanisms of MIR.

microRNAs (miRNAs or miRs) are small (19-24 nucleotides), endogenous, non-coding RNAs that have an effect on gene expression at the transcriptional or post-transcriptional level through binding to the complementary 3'-untranslated region (3'-UTR) of their target mRNAs (9-11).

miRNAs have been demonstrated to affect processes such as cell apoptosis, glucose and lipid metabolism, signal transduction (12). For instance, miR-146b prevents cardiomyocyte injury in myocardial $\mathrm{I} / \mathrm{R}$ by targeting mothers against decapentaplegic homolog 4 (13). miR-93 inhibits I/R-induced cardiomyocyte apoptosis by targeting phosphatase and tensin homolog (PTEN) (14). E2F1-dependent miR-421 regulates mitochondrial fragmentation and myocardial infarction by targeting PTEN-induced putative kinase 1 (15).

miR-144 has been demonstrated to be dysregulated during tumorigenesis, development, and metastasis of various cancers including lung cancer, oral squamous cell carcinoma and breast cancer (16-18), suggesting its potential role in tumor diagnosisand treatment. However, the effect of miR-144 on ischemic injury hasrarely been reported.

In the present study, the alteration of miR-144 levels was studied in a rat $I / R$ model and a cardiomyocyte hypoxia/reoxygenation $(\mathrm{H} / \mathrm{R})$ model, and it was observed that miR-144 was notably downregulated in $I / R$ and $H / R$. Overexpression of miR-144 constrained the infarction size and myocardial apoptosis that results from I/R. In addition, miR-144 inhibited apoptosis in cardiomyocytes under H/R treatment. Furthermore, miR-144 exerted this protective effect through regulation of apoptotic gene expression.

\section{Materials and methods}

Cell culture and transfection. H9c2 cells were obtained from the American Type Culture Collection (Manassas, VA, USA). 
The cells were cultured in Dulbecco's modified Eagle's medium (DMEM; Gibco; Thermo Fisher Scientific, Inc., Waltham, MA, USA) with $10 \%$ fetal bovine serum (Gibco; Thermo Fisher Scientific, Inc.), $100 \mathrm{U} / \mathrm{ml}$ penicillin, $100 \mu \mathrm{g} / \mathrm{ml}$ streptomycin and $110 \mathrm{mg} / \mathrm{ml}$ sodium pyruvate under a humidified air condition of $5 \% \mathrm{CO}_{2}$ at $37^{\circ} \mathrm{C}$. A total of $\sim 3 \times 10^{5}$ cells were seeded into each well of a 12-well plate, incubated overnight, and subsequently transfected with $200 \mathrm{nM}$ miR-144 mimics (Forward, TACAGT ATAGATGATGTACT; Reverse, AGTACATCATCTATACTG TA) and matched scrambled control (Forward, ATCATGCGT AGCTGACGTGA; Reverse, TCACGTCAGCTACGCATG AT) (Shanghai GenePharma Co., Ltd., Shanghai, China) using Lipofectamine ${ }^{\circledR} 2000$ (Invitrogen; Thermo Fisher Scientific, Inc.) according to the manufacturer'sprotocol. In the rescue experiment part, cells were transfected with $200 \mathrm{nM}$ miR-144 mimic or scramble together with $300 \mathrm{nM}$ FOXO1 overexpression vector or negative control vector.

In vivo gene transfer in the rat model of $I / R$ injury. A total of 24 Sprague-Dawley male rats (weight: 250-300 g; age, 8-10 weeks) were obtained from the Animal Center of Central South University (Changsha, China) and housed in an animal holding facility under standard light (12-h light/dark cycle), temperature $\left(22.0 \pm 0.5^{\circ} \mathrm{C}\right)$ and humidity $(55-60 \%)$. Rats received standard chow and water ad libitum. The present study was approved by the Renmin Hospital of Wuhan University Animal Care and Use Committee (Wuhan, China). Rats were randomly divided into four different groups (each, $n=6$ ): Sham (served as controls), I/R, I/R+miR-144 and miR-144 treatment groups. Rats were anesthetized with $40 \mathrm{mg} / \mathrm{kg}$ pentobarbital (Sigma-Aldrich; Merck KGaA, Darmstadt, Germany) intraperitoneally and placed on a temperature-controlled heating pad and a left thoracotomy was performed in the fourth intercostal space. A $100-\mu 1$ solution of adenovirus (Ad)-miR-144 with DMEM

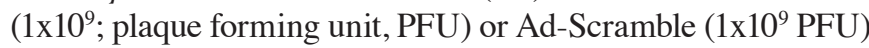
were injected into six sites on the left ventricular anterior wall. The chest cavity was closed following this administration, and the rats were allowed to recover. Three days later, all rats were anesthetized again with $40 \mathrm{mg} / \mathrm{kg}$ pentobarbital and ventilated artificially. Subsequently, the left anterior descending coronary artery (LAD) was exposed, and 6-0 silk sutures were used to ligate the LAD. Cyanosis in the anterior ventricular wall was used to confirm the ligation. Ischemia was induced using a small vinyl tube that was threaded through the ligature. Following $30 \mathrm{~min}$ of ischemia, tubes were translocated, and coronary circulation was restored for $2 \mathrm{~h}$. Subsequently, the infarct area of myocardium tissues and the blood samples were collected for the following analysis. Sham control animals were subjected to the entire surgical procedure without LAD ligation.

H/R model. At $48 \mathrm{~h}$ following transfection with miR-144 mimic or scramble, H9c2 cell hypoxia was induced by treating the cells in a modular incubator (Forma; Thermo Fisher Scientific, Inc.) in an atmosphere containing $1 \% \mathrm{O}_{2}, 94 \% \mathrm{~N}_{2}$ and $5 \% \mathrm{CO}_{2}$ for $24 \mathrm{~h}$ at $37^{\circ} \mathrm{C}$. Subsequently, the cells were incubated for $3 \mathrm{~h}$ in DMEM with $10 \% \mathrm{FBS}$ at $37^{\circ} \mathrm{C}$ in an atmosphere containing $5 \% \mathrm{CO}_{2}$.

Infarct size determination. Evans blue/triphenyltetrazolium chloride (TTC) was used to stain and measure the myocardial infarct size. Immediately after reperfusion ended, Evans blue dye solution ( $3 \mathrm{ml}, 2 \% \mathrm{wt} / \mathrm{vol}$ ) was injected into the left ventricle to identify areas at risk (AARs) at room temperature for $2 \mathrm{~h}$. Immediately following reperfusion, rats were sacrificed via exsanguination which were anesthetized as aforementioned. The hearts were harvested and were frozen for $30 \mathrm{~min}$ at $-20^{\circ} \mathrm{C}$. Subsequently, the left ventricle was sliced transversely at a thickness of $1 \mathrm{~mm}$. These slices were immediately injected with $1 \%$ TTC (Sigma-Aldrich; Merck KGaA) to detect ischemic and infarcted tissue at $37^{\circ} \mathrm{C}$. The infarcted areas are those that were not stained and were regarded as non-viable, whereas the non-infarcted areas that were stained were regarded as viable. The AAR and infarct size were measured using image J 1.48 software (National Institutes of Health, Bethesda, MD, USA).

LDH (lactate dehydrogenase) and CK (creatine kinase) activity assay. Following reperfusion, blood was obtained from the carotid artery of rats, and stored at room temperature for $30 \mathrm{~min}$. Serum was subsequently collected by centrifugation at $5,000 \mathrm{x} \mathrm{g}$ for $10 \mathrm{~min}$ at $4^{\circ} \mathrm{C}$. CK (cat. no. A032) and (cat. no. A020-1) LDH assay kits (Nanjing Jiancheng Bioengineering Institute, Nanjing, China) were used to measure activity of $\mathrm{CK}$ and $\mathrm{LDH}$ according to the manufacturer's protocols.

Terminal deoxynucleotidyl-transferase-mediated dUTP nick end labeling (TUNEL) assay. TUNEL staining was used to evaluate cardiomyocyte apoptosis. Heart tissue fixed in $4 \%$ formaldehyde at room temperature for $24 \mathrm{~h}$ and was cut into 4- $\mu \mathrm{m}$ slices following washing, dehydrating, and immersing in paraffin. The apoptotic cardiomyocytes were detected using an In situ cell death detection kit (Roche Diagnostics, Basel, Switzerland). Cells with brown stained nuclei indicated apoptotic cells and were counted in 5 microscopic fields of view under fluorescence microscopy. The ratio of TUNEL positive cells to total cardiomyocytes was calculated.

Reverse transcription-quantitative polymerase chain reaction $(R T-q P C R)$. Total RNA was extracted from $\mathrm{H} 9 \mathrm{c} 2$ cells and rat myocardium using TriZOL reagent (Invitrogen; Thermo Fisher Scientific, Inc.) according to the manufacturer'sprotocol. The cDNA was synthesized using $1 \mu \mathrm{l}$ reverse transcriptase, $2 \mu \mathrm{l}$ dNTPs, $5 \mu$ l buffer (TAKARA BIO INC, Dalian, China), a stem-loop RT primer (Invitrogen; Thermo Fisher Scientific, Inc.) and $2 \mu \mathrm{g}$ RNA, the temperature protocol was as follows: $42^{\circ} \mathrm{C}$ for $30 \mathrm{~min}$ and $85^{\circ} \mathrm{C}$ for $5 \mathrm{~min}$. The relative amount of miRNA was detected using a SYBR Green Realtime PCR Master Mix (Takara Biotechnology Co., Ltd., Dalian, China). The primers utilized were as follows: MiR-144, forward: CCT CGCACCTGGAGGCTGGCTG reverse: TTATCAGTTGGG AAAATAGTTA; U6, forward: CTCGCTTCGGCAGCACA reverse: AACGCTTCACGAATTTGCGT. The thermocycling conditions consisted of an initial, single cycle of $2 \mathrm{~min}$ at $94^{\circ} \mathrm{C}$, followed by 40 cycles of $15 \mathrm{sec}$ at $94^{\circ} \mathrm{C}, 20 \mathrm{sec}$ at $63^{\circ} \mathrm{C}$ and $30 \mathrm{sec}$ at $68^{\circ} \mathrm{C}$. The $2^{-\Delta \Delta \mathrm{Cq}}$ method (19) was utilized for measuring expression levels, and the U6 small nuclear RNA was used as an internal reference.

Western blot analysis. Liquid nitrogen was used to freeze tissue samples, and protein was extracted following lysis 
A

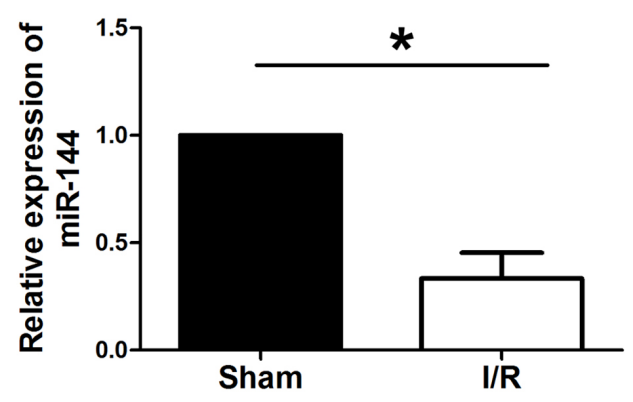

B

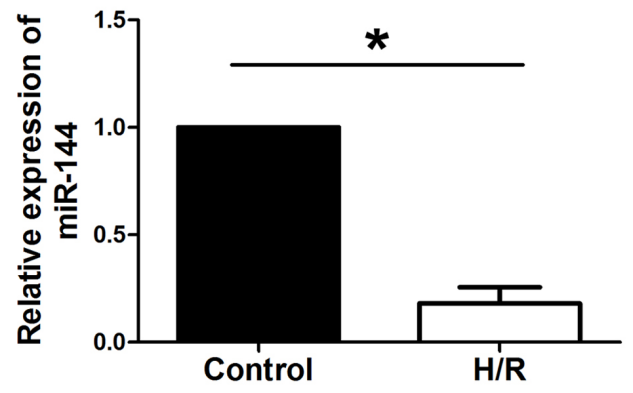

Figure 1. miR-144 is downregulated in I/R myocardium and cardiomyocytes under H/R. Reverse transcription-quantitative polymerase chain reaction was used to determine the expression of miR-144 in the (A) myocardiumfollowing $\mathrm{I} / \mathrm{R}(\mathrm{F}=61.862 ; \mathrm{df}=1)$ and in $(\mathrm{B})$ cardiomyocytes under $\mathrm{H} / \mathrm{R}(\mathrm{F}=28.182$, $\mathrm{df}=1)$. Data are presented as the mean \pm standard error of the mean $(n=6)$. "P $<0.01$. miR, microRNA; I/R, ischemia/reperfusion; H/R, hypoxia/reoxygenation; df, degrees of freedom.

A

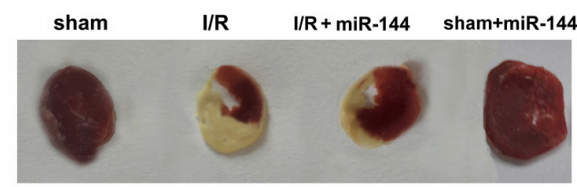

C

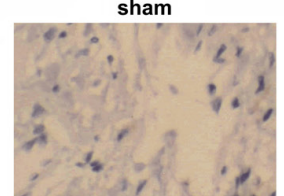

$\mathrm{I} / \mathrm{R}+\mathrm{miR}-144$

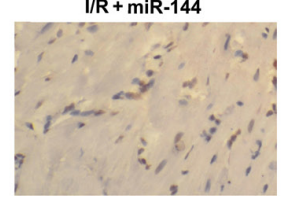

I/R

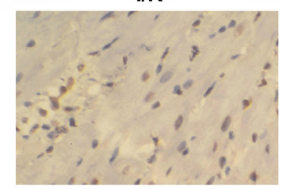

sham+miR-144

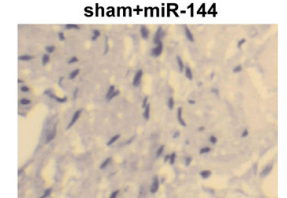

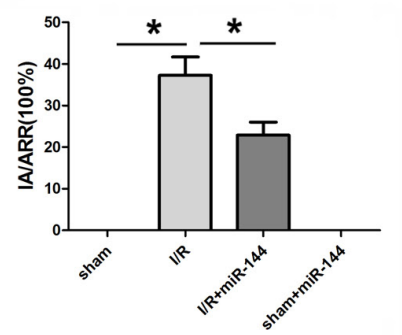

D

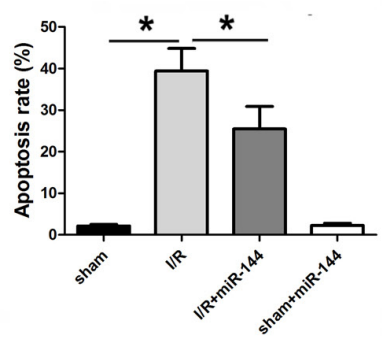

E

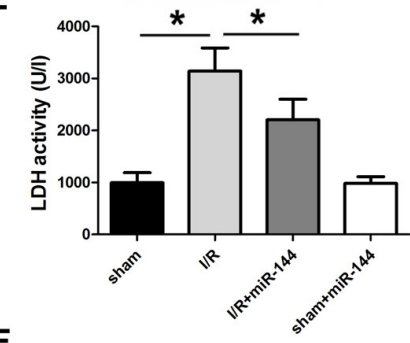

$\mathbf{F}$

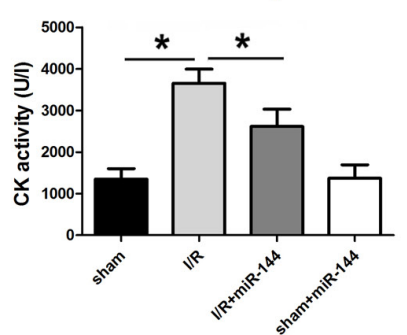

Figure 2. miR-144 induces a cardioprotective effect in vivo. (A) TTC and Evans blue-stained heart slices from the sample. (B) Infarct size was measured by TTC and Evans blue staining ( $\mathrm{F}=167.992$, $\mathrm{df}=3$ ). (C) The TUNEL assay was applied to evaluate the apoptosis in the myocardium (magnification, $\mathrm{x} 200$ ). (D) Percentage of TUNEL-positive-stained cardiomyocytes was calculated to determine apoptosis rate (F=92.326, df=3). (E) LDH activity in serum was measured $(\mathrm{F}=148.563, \mathrm{df}=3)$. $(\mathrm{F}) \mathrm{CK}$ activity in serum was measured $(\mathrm{F}=65.462, \mathrm{df}=3)$. Data are presented as the mean \pm standard error of the mean $(\mathrm{n}=6)$. ${ }^{*} \mathrm{P}<0.01$. miR, microRNA; TTC, triphenyltetrazolium chloride; TUNEL, terminal deoxynucleotidyl-transferase-mediated dUTP nick end labeling; LDH, lactate dehydrogenase; CK, creatine kinase; df, degrees of freedom; I/R, ischemia/reperfusion.

with RIPA lysis buffer (BioTeke Corporation, Beijing, China) containing $1 \mathrm{mM}$ phenylmethylsulfonyl fluoride. A bicinchoninic acid assay kit (Beyotime Institute of Biotechnology, Haimen, China) was used to evaluate the concentration of protein. Subsequently, $30 \mu \mathrm{g}$ protein was separated by $10 \%$ SDS-PAGE and electrophoretically transferred to polyvinylidene fluoride membranes. The membranes were blocked in 5\% non-fat milk in TBSTween-20 buffer (100 mM NaCl, 10 mM Tris-HCl; pH 7.4; 0.1\% Tween-20) for $2 \mathrm{~h}$ at room temperature. Subsequently, the membranes were incubated at $4{ }^{\circ} \mathrm{C}$ overnight with primary antibodies (all 1:200; Abcam, Cambridge, UK) against forkhead box protein O1 (FOXO1; cat. no. ab39670), Bcl-2 associated X protein (Bax; cat. no. ab182733), B-cell lymphoma 2 (bcl-2; cat. no. ab196495), cytochrome $c$ (cat. no. ab18738), caspase9 (cat. no. ab184786), caspase3 (cat. no. ab32042) or GAPDH (cat. no. ab181602). After washing with PBS, the membranes were incubated with horseradish peroxidase-conjugated secondary antibody for $2 \mathrm{~h}$ at room temperature. Protein bands were detected with an enhanced chemiluminescence detection kit (cat. no. 631701; Pierce; Thermo Fisher Scientific, Inc.) and ImageJ software 1.48 (National Institutes of Health).

Flow cytometry. Cell apoptosis was evaluated using a flow cytometer (BD Biosciences, Franklin Lakes, NJ, USA) and Annexin V-FITC Apoptosis Detection kit to determine the percentage of apoptotic cells. A total of $\sim 10^{6}$ cells were suspended in $200 \mu \mathrm{l}$ binding buffer, and $10 \mu \mathrm{l}$ Annexin V-fluorescein isothiocyanate and $5 \mu \mathrm{l}$ propidium iodide (all BD Biosciences) were added to the cells and subsequently incubated in the dark for $30 \mathrm{~min}$ at room temperature. Cell apoptosis was evaluated using flowjo 7.6.1 software (BD Biosciences).

Bioinformatics analysis. To elucidate the mechanism by which miR-144 inhibits I/R injury, two publicly available bioinformatics tools, miRanda (www.microrna.org/) and TargetScan 
A

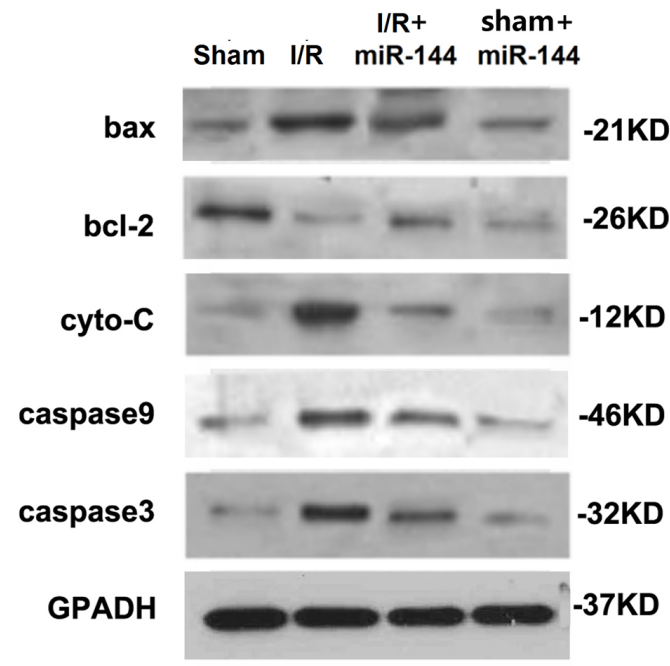

D

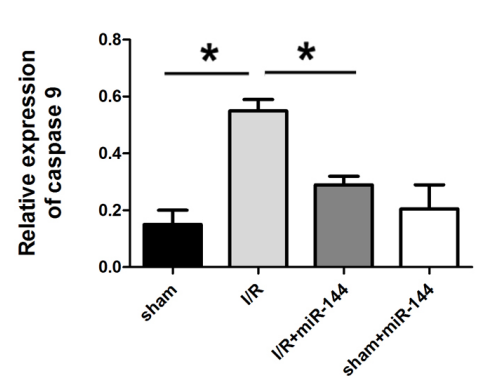

$\mathbf{E}$

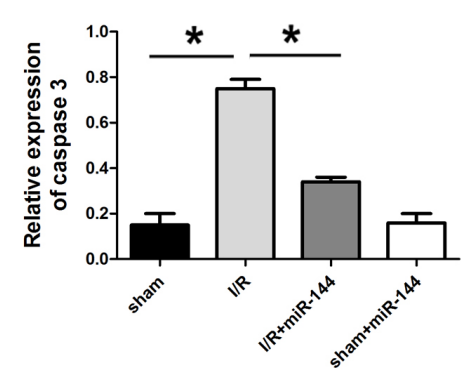

$\mathbf{F}$

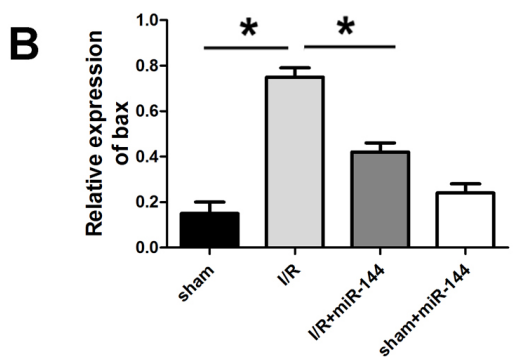

C
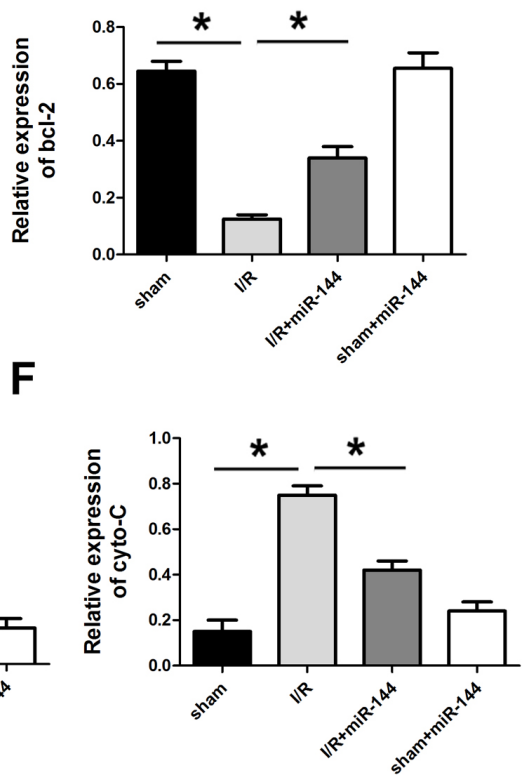

Figure 3. miR-144 regulated apoptotic protein expression. (A) Western blot analysis showing the relative apoptotic protein levels including (B) Bax (F=32.352, $\mathrm{df}=3$ ), (C) Bcl-2 ( $\mathrm{F}=22.374, \mathrm{df}=3)$, (D) caspase-9 ( $\mathrm{F}=28.542$, df=3), (E) caspase-3 ( $\mathrm{F}=20.347, \mathrm{df}=3$ ) and ( $\mathrm{F})$ cyto-C ( $\mathrm{F}=39.458$, df=3) in the myocardium. Data are presented as the mean \pm standard error of the mean $(\mathrm{n}=6)$. "P<0.01. miR, microRNA; Bcl-2, B cell lymphoma-2; Bax, Bcl-2 associated $\mathrm{X}$ protein; cyto-C, cytochrome $c ; \mathrm{df}$, degrees of freedom, $\mathrm{I} / \mathrm{R}$, ischemia reperfusion.

(http://www.targetscan.org/vert_71/), were searched for genes containing potential binding sites for miR-144 in their 3'-UTRs.

Dual-luciferase reporter assay. The putative wild and mutant miR-144 binding sequence from the 3'UTR segment of FOXO1 mRNA was cloned into the p-GL3-basic luciferase reporter plasmid (Hanbio Biotechnology Co., Ltd., Shanghai, China). H9c2 cells were transfected with $200 \mathrm{nM}$ miR-144 ASO (inhibitor of miR-144; 5'-AGTACATCATCT ATACTGTA-3') or mimic with a reporter gene p-GL3-basic containing wild-type or mutated 3'UTR sequence of FOXO1 as aforementioned. At $48 \mathrm{~h}$ following transfection, the cells were harvested, and the luciferase activity was measured using a Dual Luciferase Reporter Gene Assay kit (Promega Corporation, Madison, WI, USA). Renilla luciferase activity was used to normalize the firefly luciferase intensity.

Statistical analysis. Data are presented as the mean \pm standard error of the mean. One-way analysis of variance with a post-hoc Tukey's test and two-tailed Student's t-test were utilized to analyze the differences between groups, and $\mathrm{P}<0.05$ was considered to indicate a statistically significant difference. Analysis was performed using SPSS 17.0 software (SPSS, Inc., Chicago, IL, USA). All experiments were performed in triplicate.

\section{Results}

miR-144 is downregulated in I/R and H/R. RT-qPCR was used to evaluate the expression levels of miR-144 in I/R myocardium and in $\mathrm{H} 9 \mathrm{c} 2$ cells subjected to $\mathrm{H} / \mathrm{R}$. As presented in Fig. 1, miR-144 was significantly downregulated in myocardial $\mathrm{I} / \mathrm{R}$ rats comparedwith sham rats and in $\mathrm{H} 9 \mathrm{c} 2$ cells subjected to $\mathrm{H} / \mathrm{R}$ compared with control group cells.

miR-144 reduces myocardial infarct size and apoptosis in $I / R$ rats. To explore the importance of miR-144 in myocardial I/R injury, the adenovirus-mediated miR-144 transfer was performed via injection into six sites in the left ventricular anterior wall of the rats. The infarct area of hearts was measured using Evans blue and TTC staining. The ischemic area (IA)/AAR ratio was markedlyincreased in the I/R group compared with the sham group, demonstrating the success of I/R establishment (Fig. 2A). Overexpression of miR-144 following ischemia treatment significantly reduced the IA/AAR comparedwith the sham group (Fig. 2A and B). TUNEL staining was performed in cardiac isografts from each experimental group. Compared with the I/R group, Ad-miR-144 administration significantly reduced the number of TUNEL-positive cardiomyocytes (Fig. 2C and D). In addition, CK and LDH activity were measured, which indicates oxidative stress-induced injury. 
A
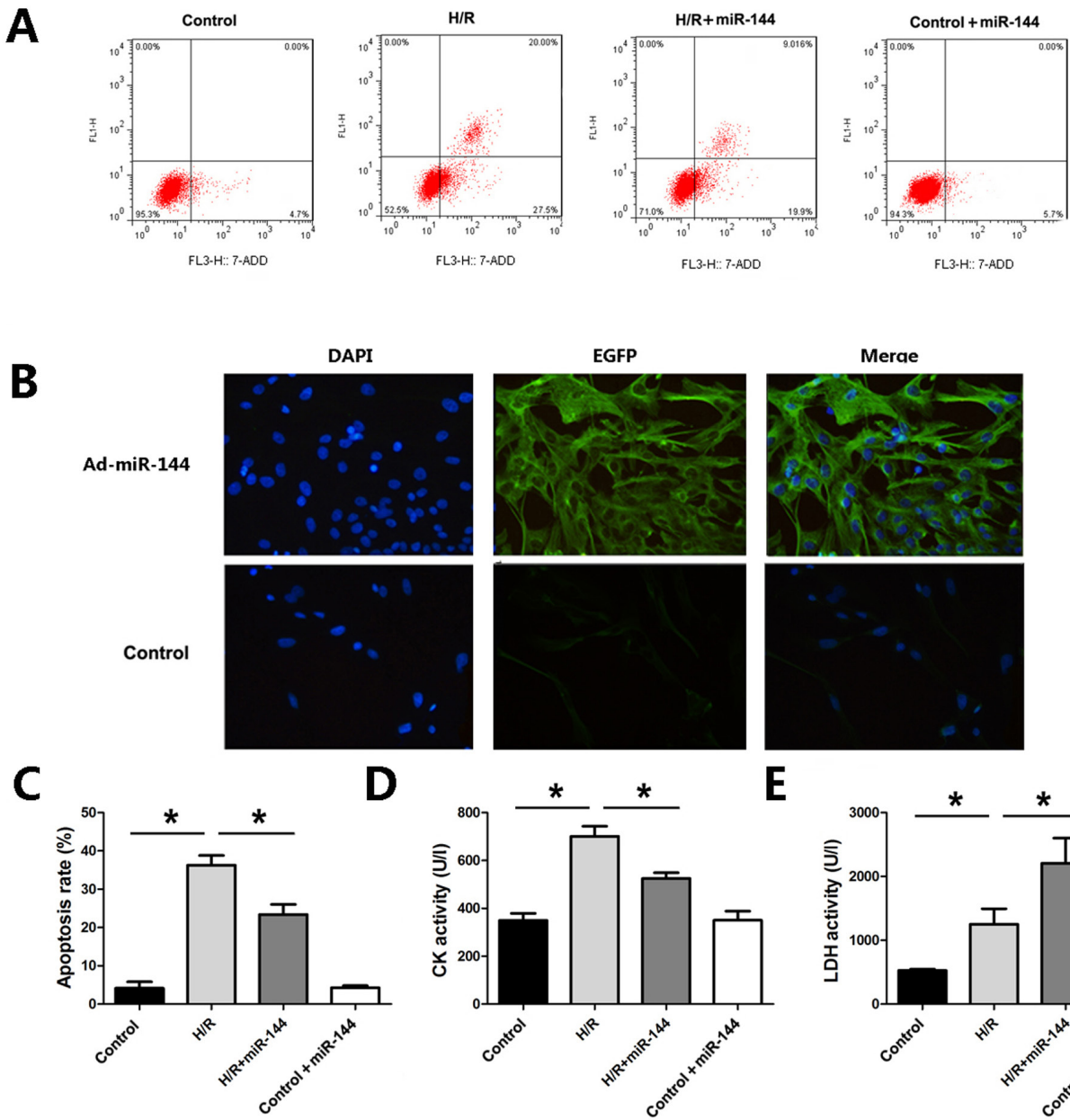

$\mathbf{E}$

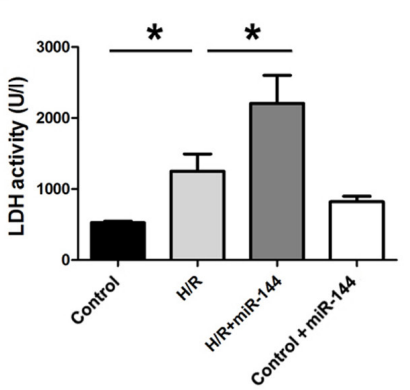

Figure 4. miR-144 induced a cardioprotective effect in vitro. (A) Flow cytometry was used to detect apoptosis in cardiomyocytes (magnification, x200), (B) Fluorescence microscopy was used to detect the infection efficiency of Ad-miR-144. (C) Quantitative analysis of apoptotic rate evaluated by flow cytometry $(\mathrm{F}=58.108, \mathrm{df}=3)$. (D) $\mathrm{CK}$ activity was measured in the cellular supernatant $(\mathrm{F}=32.178, \mathrm{df}=3)$. (E) LDH activity was measured in the cellular supernatant $(\mathrm{F}=20.320, \mathrm{df}=3)$. Data are presented as the mean \pm standard error of the mean $(\mathrm{n}=6) .{ }^{*} \mathrm{P}<0.01$. miR, microRNA; df, degrees of freedom; H/R, hypoxia/reoxygenation; $\mathrm{CK}$, creatine kinase; $\mathrm{LDH}$, lactate dehydrogenase.

Overexpression of miR-144 resulted in significantly decreased activity of CK and LDH compared with the I/R group (Fig. 2E and F).

miR-144 regulates apoptotic protein expression. The expression of apoptosis-related proteins was subsequently assessed. Compared with the sham group, the anti-apoptotic protein $\mathrm{Bcl}-2$ was downregulated in the I/R group and apoptotic proteins, Bax, cytochrome $c$, caspase 9 and caspase 3 , were all significantly upregulated in the I/R group (Fig. 3). To explore whether miR-144 administration without $\mathrm{I} / \mathrm{R}$ treatment would lead to the changes mentioned above, Ad-miR-144 was administered following sham treatment. The results revealed that miR-144 administration in the absence of $\mathrm{I} / \mathrm{R}$ did not have any significant effect on the IA/AAR, myocardial apoptosis, CK and LDH activity or apoptotic proteins expression, in comparison with the sham group (Figs. 1-3).

miR-144 attenuates H9c2 cell apoptosis. To further evaluate the effect of miR-144 on myocardial infarction protection, an $\mathrm{H} / \mathrm{R}$ model was established in $\mathrm{H} 9 \mathrm{c} 2$ cells. Flow cytometry was used to assess apoptosis in H9c2 cells. As presented in
Fig. 4A, induction of $\mathrm{H} / \mathrm{R}$ resulted in a marked increase in apoptosis in H9c2 cells, whereas Ad-miR-144 treatment decreased the apoptosis rates in comparison with the H/R group (Fig. 4A and B). Furthermore, miR-144 overexpression significantly attenuated $\mathrm{CK}$ and LDH activities, which were significantly elevated by the H/R treatment, as compared to the control group (Fig. 4C-E). To explore whether miR-144 administration without $\mathrm{H} / \mathrm{R}$ treatment would lead to any of these findings, Ad-miR-144 was administered in the control $\mathrm{H} 9 \mathrm{c} 2$ cells. The results revealed that miR-144 administration did not result in any significant changes in the cell apoptosis, CK or LDH activity in the absence of H/R, compared with the control group.

miR-144 directly targets FOXO1. The levels of FOXO1 were analyzed by MiRanda and TargetScan, and the results indicated that FOXO1 is targeted by miR-144. The 3'-UTR of the FOXO1 mRNA has a binding site for miR-144. To verify this binding, the putative miR-144 binding sequence from the 3'UTR segment of FOXO1 mRNA was cloned into the luciferase reporter plasmid. An additional luciferase reporter vector was constructed with a mutation in the miR-144 binding site (Fig. 5A). H9c2 cells were co-transfected with miR-144 mimics or negative control miRNA 
A

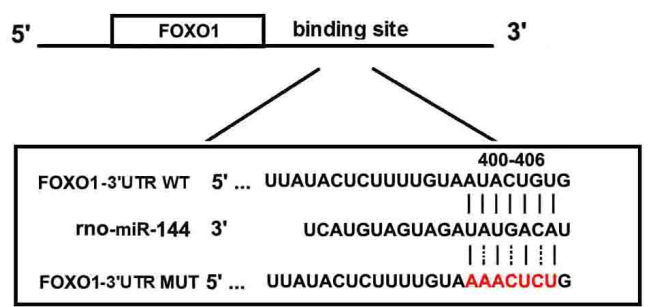

C

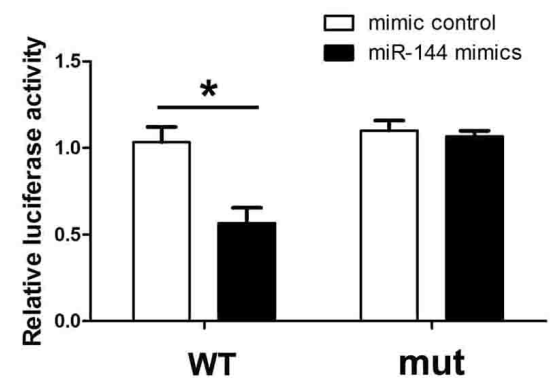

B

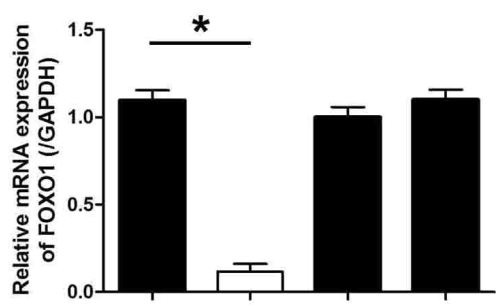

D

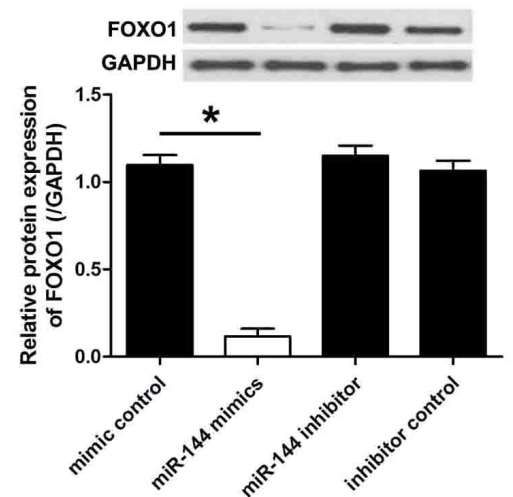

Figure 5. miR-144 targets FOXO1 in cardiomyocytes. (A) Conserved binding sites of miR-144 on the WT and mut3'-UTR of FOXO1 in rats. (B) A luciferase reporter construct with the WT or mut miR-144 binding sequence from the 3'-UTR of the FOXO1 was transfected into cardiomyocytes with miR-144 mimics and mimic control $(\mathrm{F}=21.128, \mathrm{df}=3)$. (C) The mRNA level of FOXO1 was detected by reverse transcription-quantitative polymerase chain reaction ( $\mathrm{F}=18.125$, $\mathrm{df}=3)$. (D) The protein level of FOXO1 was detected by western blot analysis $(\mathrm{F}=18.248, \mathrm{df}=3)$. Data are presented as the mean \pm standard error of the mean $(\mathrm{n}=6)$. ${ }^{\text {P }}<0.01$. miR, microRNA; FOXO1, forkhead box protein O1; WT, wild-type; mut, mutant; UTR, untranslated region; df, degrees of freedom.

A

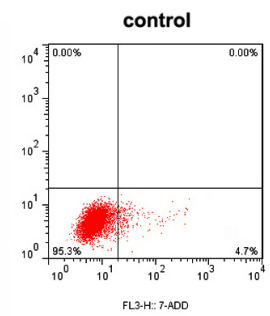

B

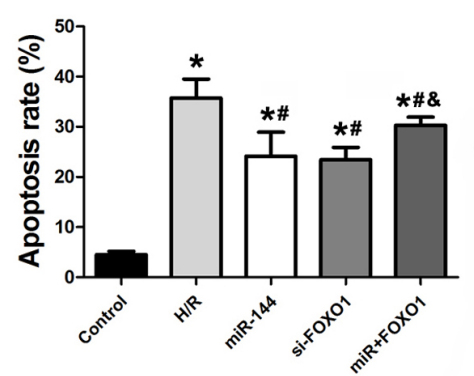

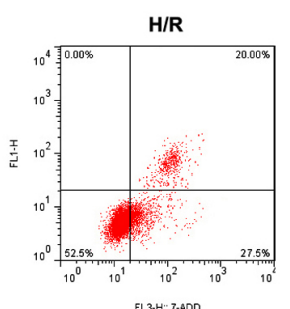

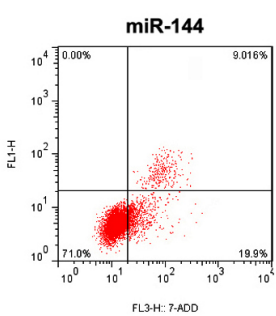

C

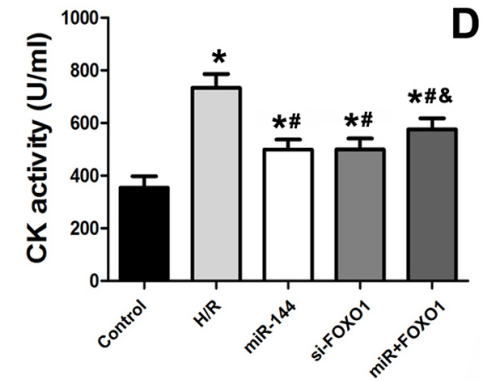

si-FOX01

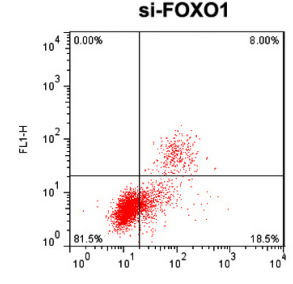

Figure 6. FOXO1 is a target gene that is associated with miR-144-induced cardioprotective effects in vitro. (A and B) Flow cytometry was used to detect apoptosis in cardiomyocytes $(\mathrm{F}=27.952, \mathrm{df}=4)$. $(\mathrm{C}) \mathrm{CK}$ activity was measured in the cellular supernatant $(\mathrm{F}=41.157, \mathrm{df}=4)$. ( $\mathrm{D}) \mathrm{LDH}$ activity was measured in the cellular supernatant $(\mathrm{F}=32.362, \mathrm{df}=4)$. Data are presented as the mean \pm standard error of the mean $(\mathrm{n}=6)$. ${ }^{*} \mathrm{P}<0.01 \mathrm{vs}$. control; ${ }^{\#} \mathrm{P}<0.01 \mathrm{vs}$. $\mathrm{H} / \mathrm{R}$ group; ${ }^{\&} \mathrm{P}<0.01$ vs. miR-144 group. FOXO1, forkhead box protein O1; miR, microRNA; df, degrees of freedom; CK, creatine kinase; LDH, lactate dehydrogenase.

for $48 \mathrm{~h}$, and luciferase activity was subsequently measured. It was observed that miR-144 mimics significantly inhibited luciferase activity whereas the negative control miRNA did not (Fig. 5B). Both miR-144 and negative control did not change the luciferase activity when co-transfected with mutant FOXO1. To demonstrate that miR-144 targets FOXO1 and inhibits endogenous FOXO1 expression, the protein levels of FOXO1 were measured in H9c2 cells transfected with miR-144 mimics or the negative control. As presented in Fig. 5C and D, transfection with miR-144 mimics significantly downregulated the mRNA and protein levels of FOXO1.

FOXO1 overexpression reverses the effect of miR-144 on cardiomyocyte injury. To demonstrate the assumption that 
A

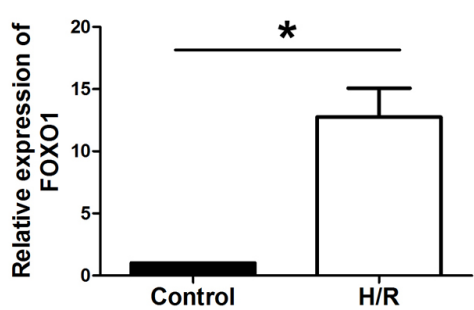

B

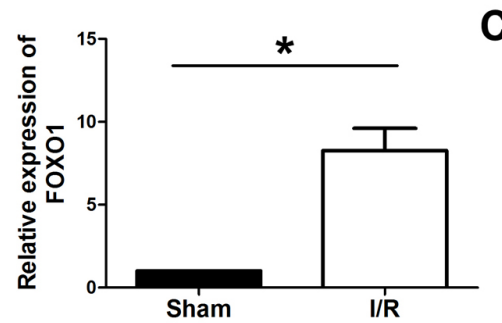

C

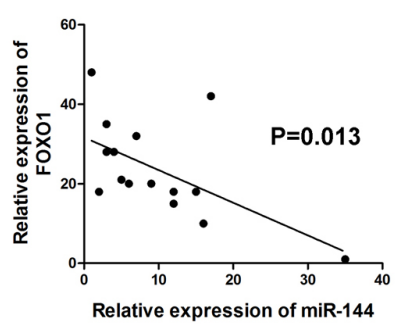

Figure 7. Correlation between miR-144 and FOXO1. Reverse transcription-quantitative polymerase chain reactionwas used to determine the expression of FOXO1 levels in the (A) myocardiumfollowing $\mathrm{I} / \mathrm{R}(\mathrm{F}=81.859, \mathrm{df}=1)$ and in $(\mathrm{B})$ cardiomyocytes under $\mathrm{H} / \mathrm{R}(\mathrm{F}=71.954, \mathrm{df}=1)$. $(\mathrm{C}) \mathrm{Pearson}$ analysis was performed to evaluate the correlation between miR-144 and FOXO1. Data are presented as the mean \pm standard error of the mean $(n=6)$. ${ }^{*}<0.01$. miR, microRNA; FOXO1, forkhead box protein O1; df, degrees of freedom; H/R, hypoxia/reoxygenation; I/R, ischemia/reperfusion.

miR-144 targets FOXO1, cardiomyocytes were transfected with $100 \mathrm{nM}$ miR-144 mimics and a FOXO1 overexpression vector to ectopically increase miR-144 and FOXO1 levels. Overexpression of FOXO1 significantly reversed the apoptosis-inhibiting effect of miR-144 (Fig. 6A and B). In addition, the activity of CK and LDH were measured in these cells. Consistently, FOXO1 overexpression reversed the significantly increased activities of $\mathrm{CK}$ and $\mathrm{LDH}$ when compared with the miR-144 treatment group, which indicates that expression was decreased by miR-144 (Fig. 6C and D).

FOXO1 is upregulated in myocardium and H9c2 cells, and is inversely correlated with miR-144 expression. RT-qPCR was performed to determine the expression levels of $\mathrm{FOXOI}$ in myocardium and $\mathrm{H} 9 \mathrm{c} 2$ cells. The results demonstrated that FOXO1 was significantly upregulated in theH9c2 cells subjected to $\mathrm{H} / \mathrm{R}$ and $\mathrm{I} / \mathrm{R}$ myocardium, compared with the control and sham groups, respectively (Fig. 7A and B). In addition, Pearson analysis was performed to evaluate the correlation between miR-144 and FOXO1. It was observed that the expression level of $\mathrm{FOXO1}$ was inversely correlated with the expression level of miR-144 in myocardium tissues (Fig. 7C).

\section{Discussion}

Previous studies have identified miR-144 as a circulating effector of rIPC-induced cardioprotection (20). Upregulation of the miR-144/451 cluster is correlated with cardioprotection against hypoxic stress through the CUGBP, Elav-like family member 2-cyclooxygenase-2 signal pathway (21). These findings have indicated the potential role of miR-144 in cardioprotection.

In the present study, miR-144 expression levels were measured in ischemic hearts using a well-established I/R model in vivo and in vitro. It was observed that miR-144 was dysregulated. In addition, overexpression of miR-144 reduced the infarct size and cardiomyocyte apoptosis, whereas depletion of miR-144 increased the sensitivity to I/R in cell apoptosis. These results confirmed that miR-144 was a positive regulator of cardioprotection following $\mathrm{I} / \mathrm{R}$ injury.

Apoptosis is a type of cell death that is associated with morphological problems and is significantly associated with nuclear pyknosis and karyorhexis (22). Cardiomyocyte apoptosis serves a key role in ischemic injuries. Increasing evidence has indicated that apoptosis caused by $\mathrm{I} / \mathrm{R}$ results in myocyte injury and that suppressing cardiomyocyte apoptosis leads to cardioprotection against I/R injury $(23,24)$. miR-144 has primarily been reported to affect the apoptosis process in human cancers. For instance, miR-144-3p has been reported to lead to apoptosis inhuman salivary adenoid carcinoma cells by targeting mechanistic target of rapamycin (14). In addition, miR-144 is able to induce apoptosis and autophagy in lung cancer cells by targeting TP53-inducible glycolysis and apoptosis regulator, and zinc finger X-linked, and in glioblastoma by targeting c-Met (15-17).

miRNAs elicit their function via post-transcriptional regulation of target mRNAs. Computational analysis was utilized to predict the potential gene targets. miR-144 was predicted to target various genes in many other kinds of cells $(25,26)$. However, these predicted targets must be experimentally verified as gene expression, as well as cellular functions, have specificity in different cell types. In the present study, FOXO1 was predicted to be a target of miR-144 in cardiomyocytes. As predicted, a luciferase activity assay verified that miR-144 directly targeted FOXO1.

FOXO1 is an important forkhead transcription factor, which is influential in the regulation of cellular metabolism, proliferation, and cell death (27). Hosaka et al (28) previously reported that the FOXO1 gene affects the development of the cardiovascular system. It was also reported that FOXO1 mediates cardiomyocyte apoptosis by activating the expression of inducible nitric oxide synthase $(29,30)$. In the cardiovascular system, sirtuin 1 may protect the ischemia-stressed cardiomyocytes from apoptosis by regulating FOXO1 (31). Similarly, any defect of FOXO1 that exists in mouse cardiomyocytes may contribute to decreased myocardial function following acute $\mathrm{I} / \mathrm{R}$ by increasing oxidative damage (26). Furthermore, apelin improves cardiomyocyte viability and compensates excessive mitochondria-derived ROS formation via the FOXO1 pathway in obese mice (18). FOXO1 was reported to be targeted by numerous miRNAs, such as miR-181a, miR-3188, miR-9 and miR-155, particularly in human cancers (32-35). The present study assessed the effect of miR-144 and FOXO1 on ischemia/reperfusion injury; however, the precise mechanism of this effect was not elucidated. Thus, it may be necessary to perform transgenic animals studies in the future.

It was demonstrated in the present study that miR-144, a significantly upregulated miRNA in H/R myocardial cells, 
serves a crucial role in $\mathrm{H} / \mathrm{R}$ apoptosis in myocardial cells by regulating FOXO1. Based on these findings, it appears that miR-144 may serve as a promising target for the prevention of myocardial I/R injury, although further study of the in vivo effect is required to fully elucidate the functional consequences of this miRNA.

\section{Acknowledgements}

Not applicable.

\section{Funding}

Funding was provided by the Natural Science Foundation of Inner Mongolia autonomous region (funding no. 2016MS0867).

\section{Availability of data and materials}

The analyzed data sets generated during the present study are available from the corresponding author on reasonable request.

\section{Authors' contributions}

LE contributed to the completement of the experiments and data analysis. HJ was a contributor in analysing the data and writting the manuscript. ZL contributed to the experimental design and manuscript revision.

\section{Ethics approval and consent to participate}

The present study was approved by the Renmin Hospital of Wuhan University Animal Care and Use Committee (Wuhan, China).

\section{Consent for publication}

Not applicable.

\section{Competing interests}

The authors declare that they have no competing interests.

\section{References}

1. Carden DL and Granger DN: Pathophysiology of ischaemiareperfusion injury. J Pathol 190: 255-266, 2000.

2. Dorweiler B, Pruefer D, Andrasi TB, Maksan SM, Schmiedt W, Neufang A and Vahl CF: Ischemia-reperfusion injury: Pathophysiology and clinical implications. Eur J Trauma Emerg Surg 33: 600-612, 2007.

3. Wang JX, Zhang XJ, Li Q, Wang K, Wang Y, Jiao JQ, Feng C, Teng S, Zhou LY, Gong Y, et al: MicroRNA-103/107 regulate programmed necrosis and myocardial ischemia/reperfusion injury through targeting FADD. Circ Res 117: 352-363, 2015.

4. Li Y, Wen S, Yao X, Liu W, Shen J, Deng W, Tang J, Li C and Liu K: MicroRNA-378 protects against intestinal ischemia/reperfusion injury via a mechanism involving the inhibition of intestinal mucosal cell apoptosis. Cell Death Dis 8: e3127, 2017.

5. Kang B, Li W, Xi W, Yi Y, Ciren Y, Shen H, Zhang Y, Jiang H, Xiao J and Wang Z: Hydrogen sulfide protects cardiomyocytes against apoptosis in ischemia/reperfusion through MiR-1-regulated histone deacetylase 4 pathway. Cell Physiol Biochem 41: 10-21, 2017
6. Hausenloy DJ and Yellon DM: Myocardial ischemia-reperfusion injury: A neglected therapeutic target. J Clin Invest 123: 92-100, 2013.

7. Rakotovao A, Tanguy S, Toufektsian MC, Berthonneche C, Ducros V, Tosaki A, de Leiris J and Boucher F: Selenium status as determinant of connexin-43 dephosphorylation in ex vivo ischemic/reperfused rat myocardium. J Trace Elem Med Biol 19: 43-47, 2005.

8. Barandier C, Tanguy S, Pucheu S, Boucher F and De Leiris J: Effect of antioxidant trace elements on the response of cardiac tissue to oxidative stress. Ann N Y Acad Sci 874: 138-155, 1999.

9. Valinezhad Orang A, Safaralizadeh R and Kazemzadeh-Bavili M: Mechanisms of miRNA-mediated gene regulation from common downregulation to mRNA-specific upregulation. Int J Genomics 2014: 970607, 2014.

10. Bier A, Giladi N, Kronfeld N, Lee HK, Cazacu S, Finniss S, Xiang C, Poisson L, deCarvalho AC, Slavin S, et al: MicroRNA-137 is downregulated in glioblastoma and inhibits the stemness of glioma stem cells by targeting RTVP-1. Oncotarget 4: 665-676, 2013.

11. Yang WB, Chen PH, Hsu T I, Fu TF, Su WC, Liaw H, Chang WC and Hung JJ: Sp1-mediated microRNA-182 expression regulates lung cancer progression. Oncotarget 5: 740-753, 2014.

12. Holik AK, Lieder B, Kretschy N, Somoza MM, Held S and Somoza V: $N(\varepsilon)$-Carboxymethyllysine increases the expression of miR-103/143 and enhances lipid accumulation in 3T3-L1 cells. J Cell Biochem 117: 2413-2422, 2016.

13. Di YF, Li DC, Shen YQ, Wang CL, Zhang DY, Shang AQ and $\mathrm{Hu} \mathrm{T}$ : MiR-146b protects cardiomyocytes injury in myocardial ischemia/reperfusion by targeting Smad4. Am J Transl Res 9: 656-663, 2017.

14. Ke ZP, Xu P, Shi Y and Gao AM: MicroRNA-93 inhibits ischemia-reperfusion induced cardiomyocyte apoptosis by targeting PTEN. Oncotarget 7: 28796-28805, 2016.

15. Wang K, Zhou LY, Wang JX, Wang Y, Sun T, Zhao B, Yang YJ, An T, Long B, Li N, et al: E2F1-dependent miR-421 regulates mitochondrial fragmentation and myocardial infarction by targeting Pink1. Nat Commun 6: 7619, 2015.

16. Wang S, Xu Z and Wang L: Shuanghuang Shengbai granule cures myelosuppression and suppresses lung cancer progression: Mechanism and therapeutic targets from the aspect of microRNAs. Oncotarget 8: 62154-62166, 2017.

17. Manikandan M, Deva Magendhra Rao AK, Arunkumar G, Manickavasagam M, Rajkumar KS, Rajaraman R and Munirajan AK: Oral squamous cell carcinoma: microRNA expression profiling and integrative analyses for elucidation of tumourigenesis mechanism. Mol Cancer 15: 28, 2016.

18. Madhavan D, Peng C, Wallwiener M, Zucknick M, Nees J, Schott S, Rudolph A, Riethdorf S, Trumpp A, Pantel K, et al: Circulating miRNAs with prognostic value in metastatic breast cancer and for early detection of metastasis. Carcinogenesis 37: 461-470, 2016.

19. Livak KJ and Schmittgen TD: Analysis of relative gene expression data using real-tie quantitative PCR and the 2(-Delta Delta C(T)) method. Methods 25: 402-408, 2001.

20. Li J, Rohailla S, Gelber N, Rutka J, Sabah N, Gladstone RA, Wei C, Hu P, Kharbanda RK and Redington AN: MicroRNA-144 is a circulating effector of remote ischemic preconditioning. Basic Res Cardiol 109: 423, 2014.

21. Zhang X, Wang X, Zhu H, Zhu C, Wang Y, Pu WT, Jegga AG and Fan GC: Synergistic effects of the GATA-4-mediated miR-144/451 cluster in protection against simulated ischemia/reperfusion-induced cardiomyocyte death. J Mol Cell Cardiol 49: 841-850, 2010.

22. Huang Z, Ye B, DaiZ, Wu X,LuZ, Shan Pand Huang W: Curcumin inhibits autophagy and apoptosis in hypoxia/reoxygenationinduced myocytes. Mol Med Rep 11: 4678-4684, 2015.

23. Yin Y, Guan Y, Duan J, Wei G, Zhu Y, Quan W, Guo C, Zhou D, Wang Y, Xi M and Wen A: Cardioprotective effect of Danshensu against myocardial ischemia/reperfusion injury and inhibits apoptosis of H9c2 cardiomyocytes via Akt and ERK1/2 phosphorylation. Eur J Pharmacol 699: 219-226, 2013.

24. Hadi NR, Yusif FG, Yousif M and Jaen KK: Both castration and goserelin acetate ameliorate myocardial ischemia reperfusion injury and apoptosis in male rats. ISRN Pharmacol 2014: 206951, 2014.

25. Pase L, Layton JE, Kloosterman WP, Carradice D, Waterhouse PM and Lieschke GJ: miR-451 regulates zebrafish erythroid maturation in vivo via its target gata2. Blood 113: 1794-1804, 2009. 
26. Sangokoya C, Telen MJ and Chi JT: microRNA miR-144 modulates oxidative stress tolerance and associates with anemia severity in sickle cell disease. Blood 116: 4338-4348, 2010.

27. Puthanveetil P, Wan A and Rodrigues B: FoxO1 is crucial for sustaining cardiomyocyte metabolism and cell survival. Cardiovasc Res 97: 393-403, 2013.

28. Hosaka T, Biggs WR III, Tieu D, Boyer AD, Varki NM, Cavenee WK and Arden KC: Disruption of forkhead transcription factor (FOXO) family members in mice reveals their functional diversification. Proc Natl Acad Sci USA 101: 2975-2980, 2004

29. Puthanveetil P, Wang Y, Zhang D, Wang F, Kim MS, Innis S, Pulinilkunnil T, Abrahani A and Rodrigues B: Cardiac triglyceride accumulation following acute lipid excess occurs through activation of a FoxO1-iNOS-CD36 pathway. Free Radic Biol Med 51: 352-363, 2011.

30. Puthanveetil P, Zhang D, Wang Y, Wang F, Wan A, Abrahani A and Rodrigues B: Diabetes triggers a PARP1 mediated death pathway in the heart through participation of FoxO1. J Mol Cell Cardiol 53: 677-686, 2012.

31. Chen CJ, Yu W, Fu YC, Wang X, Li JL and Wang W: Resveratrol protects cardiomyocytes from hypoxia-induced apoptosis through the SIRT1-FoxO1 pathway. Biochem Biophys Res Commun 378: 389-393, 2009.
32. Xu H, Zhu J,Hu C, Song H and Li Y: Inhibition of microRNA-181a may suppress proliferation and invasion and promote apoptosis of cervical cancer cells through the PTEN/Akt/FOXO1 pathway. J Physiol Biochem 72: 721-732, 2016.

33. Zhao M, Luo R, Liu Y, Gao L, Fu Z, Fu Q, Luo X, Chen Y, Deng X, Liang Z, et al: miR-3188 regulates nasopharyngeal carcinoma proliferation and chemosensitivity through a FOXO1-modulated positive feedback loop with mTOR-p-PI3K/AKT-c-JUN. Nat Commun 7: 11309, 2016.

34. Yan C, Chen J, Li M, Xuan W, Su D, You H, Huang Y, Chen N and Liang X: A decrease in hepatic microRNA-9 expression impairs gluconeogenesis by targeting FOXO1 in obese mice. Diabetologia 59: 1524-1532, 2016.

35. Hou L, Chen J, Zheng Y and Wu C: Critical role of miR-155/FoxO1/ROS axis in the regulation of non-small cell lung carcinomas. Tumour Biol 37: 5185-5192, 2016.

(i) $\odot$ This work is licensed under a Creative Commons Attribution-NonCommercial-NoDerivatives 4.0 International (CC BY-NC-ND 4.0) License. 